\title{
Formation Depths of Spectral Lines in Cepheids
}

\author{
Michael D. Albrow ${ }^{1}$ and P. L. Cottrell \\ Mount John University Observatory, Department of Physics and \\ Astronomy, University of Canterbury, Private Bag 4800, Christchurch \\ 8001, New Zealand
}

\section{Introduction}

There has been a number of observational programmes that have endeavoured to investigate the atmospheric velocity fields in Cepheids (e.g., Sanford 1956, Wallerstein et al. 1992, Butler 1993). These studies measured the radial velocities of lines of different strength, excitation and ionisation potential as these provide an indication of line formation at different levels in the atmosphere. From these measurements, the presence of velocity gradients can be inferred, but determination of the magnitude of such gradients requires knowledge of the spectral line depth of formation. Through dynamical modelling we are endeavouring to ascertain what is actually being measured in the above observational programmes.

\section{Contribution function}

The function giving the relative contribution of different atmospheric layers to a spectral line flux depression,

$$
C_{U}\left(\log _{10} \tau_{0}\right)=\frac{(\ln 10) \tau_{0}}{\kappa_{0}} \int_{0}^{1} \kappa_{l}\left(I_{c}-S_{l}\right) e^{-\tau / \mu} d \mu
$$

has been derived by Albrow \& Cottrell (1995). This function has optical depth as its independent variable but also changes with wavelength. The most meaningful way to display the flux contribution across a spectral line is to plot a series of contours in what is known as the 'flux contribution contour' diagram (Albrow 1994, Albrow \& Cottrell 1995).

Using 'snapshot' models of Cepheid atmospheres we have investigated the effects of velocity fields on the depths of formation of spectral lines and how radial velocities measured from these lines relate to the velocities in the model. In most cases the correlation between the atmospheric velocity and the centre of absorption is good, with the contours tending to follow the velocity line. The relationship tends to worsen as line strength is increased.

An interesting phenomenon that occurs with a changing velocity gradient is the presence of spatially separated absorption centres. These are more prevalent in stronger lines but are still present at certain phases in weak lines. In Fig. 1, for

\footnotetext{
${ }^{1}$ New Zealand Science and Technology Postdoctoral Fellow; Visiting Fellow, SAAO
} 

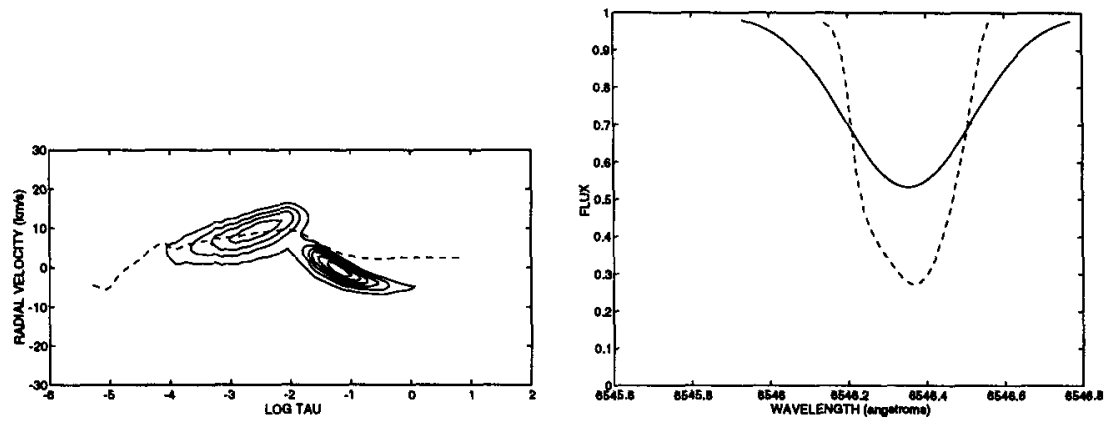

Figure 1. (a) Flux contribution contour diagram for an Fe I line with $\log g f=-2$ and $\chi=2.76 \mathrm{eV}$. The atmosphere is taken from the bump phase of a model of an 11 day Cepheid. The velocity (divided by a projection factor) is shown as a dashed line. (b) Flux profiles for the spectral line from (a). Dashed line, raw (unbroadened) flux; solid line, with radial-tangential macroturbulence $\zeta=10 \mathrm{~km} \mathrm{~s}^{-1}$.

example, the velocity near $\log \tau \sim-2.5$ (the formation depth of the core of an unshifted line) is sufficient to move the absorption at this depth by $\sim 10 \mathrm{~km} \mathrm{~s}^{-1}$ in wavelength space. The projection effect means that this shift is not the same amount across the stellar disc (more near the centre of the disc, less towards the limb) and the peak magnitude of the contribution function in the shifted 'core' is much less than if the atmosphere was static. Interior to this, near $\log \tau \sim-1$ (the formation depth of the wing of the spectral line in a static atmosphere), the projected velocity is close to zero $\left(\sim 3 \mathrm{~km} \mathrm{~s}^{-1}\right)$. The blue (negative radial velocity) wing of the spectral line will be formed as in a static atmosphere. There is little differential Doppler shift across the stellar disc to smear out the contribution function in wavelength space and the flux contribution is actually greater here than at the 'core' depth. The red (positive radial velocity) wing of the spectral line makes no contribution at this depth because it is masked by the shallower Doppler-shifted 'core' absorption. The observed (real) core of the spectral line, which appears with a radial velocity of $\sim 5 \mathrm{~km} \mathrm{~s}^{-1}$, contains contributions from the blue wing of the unshifted line and from the redshifted 'core'. The structure inherent in the line profile is unobservable because of the effects of turbulent broadening.

\section{References}

Albrow, M. D. 1994, $\mathrm{PhD}$ thesis, University of Canterbury

Albrow, M. D., \& Cottrell, P. L. 1995, MNRAS, submitted

Butler, R. P. 1993, ApJ, 415, 323

Sanford, R. F. 1956, ApJ, 123, 201

Wallerstein, G., Jacobsen, T., Cottrell, P. L., Clark, M., \& Albrow, M. 1992, MNRAS, 259, 474 